\title{
Perda de eletrólitos durante uma competição de duatlo terrestre no calor
}

CDD. 20.ed. 796.022

796.071

\author{
Geórgia Franco BECKER* \\ Liziane Maahs FLORES* \\ Cláudia Dornelles SCHNEIDER ${ }^{* * * *}$ \\ Orlando LAITANO****
}

*Escola de Educação

Física, Universidade

Federal do Rio Grande do Sul.

**Faculdade de Medicina, Universidade Federal do Rio Grande do Sul.

$* * \star$ Universidade $\mathrm{Fe}$ deral de Ciências da Saúde de Porto Alegre. $* * * *$ Universidade $\mathrm{Fe}$ deral do Vale do São Francisco.

Eventos esportivos prolongados, como o duatlo ( $6 \mathrm{~km}$ corrida, $26 \mathrm{~km}$ ciclismo e $4 \mathrm{~km}$ corrida) podem levar o atleta a um desequilibrio hidroeletrolítico, devido a perdas elevadas de suor, em especial se realizados no calor. 0 presente estudo avaliou as perdas de sódio $\left(\mathrm{Na}^{+}\right)$, potássio $\left(\mathrm{K}^{+}\right)$e cloreto $\left(\mathrm{Cl}^{-}\right)$durante uma competição de duatlo realizada no calor $\left(31,2^{\circ} \mathrm{C}\right.$ e $51 \%$ de umidade relativa do ar), analisando a composição do suor e os níveis sanguíneos destes eletrólitos. Doze atletas fizeram parte deste estudo. Coletas de sangue foram realizadas antes e após a competição, e o suor foi coletado utilizando-se adesivos especificos. 0 tempo médio para completar a competição foi de $85,0 \pm 6,57 \mathrm{~min}$. 0 percentual de desidratação foi $3,0 \pm 0,92 \%$. A reposição de líquidos perdidos durante a competição foi $31 \pm 18,7 \%$. $A$

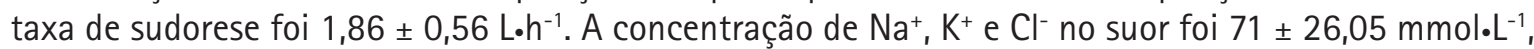
$5,43 \pm 1,98 \mathrm{mmol} \cdot \mathrm{L}^{-1}$ e $58,93 \pm 25,99 \mathrm{mmol} \cdot \mathrm{L}^{1}$, respectivamente. A perda total de $\mathrm{Na}^{+}, \mathrm{K}^{+}$e $\mathrm{Cl}^{-}$no suor foi $132,11 \pm 62,82 \mathrm{mmol}, 10,09 \pm 5,01 \mathrm{mmol}$ e 109,75 $\pm 58,49 \mathrm{mmol}$, respectivamente. Em conclusão, os atletas não ingeriram líquido suficiente para repor o volume de fluidos perdido. Além disto, os participantes apresentaram elevada taxa de sudorese acompanhada de perdas de $\mathrm{Na}^{+}, \mathrm{K}^{+}$e $\mathrm{Cl}^{-}$. Apesar disso, não ocorreram alterações nas concentrações de eletrólitos séricos.

UnITERMos: Exercício; Desidratação; Suor; Temperatura.

\section{Introdução}

O exercício quando realizado em ambiente quente leva a mudanças na capacidade do corpo em controlar seu ambiente interno devido às altas taxas de produção de calor metabólico e ganho de calor por transferência física do ambiente (MaUghan \& SHIRREFFs, 2004). Desta forma, eventos atléticos prolongados (com duração $>1$ hora) como maratonas, corridas de triatlo e duatlo, especialmente quando associado ao estresse térmico do ambiente quente, podem colocar os participantes em risco de desequilíbrio hidroeletrolítico e afetar a capacidade de termorregulação corporal.

Durante o exercício físico realizado no calor, os principais mecanismos de troca de calor são o aumento do fluxo sanguíneo cutâneo e a secreção e a evaporação do suor. A secreção de suor resulta em perda de água corporal, podendo levar à desidratação (KeNEFIK \& SAWKA, 2007). A desidratação, se ocorrer de forma significativa, pode levar à diminuição do rendimento físico e cognitivo, com consequentes implicaçôes para os resultados da competição (MAUGHAN, WATSON, Evans, BROAD \& SHIRREFFS, 2007). O suor é composto por água e eletrólitos, dos quais o sódio $\left(\mathrm{Na}^{+}\right)$, o potássio $\left(\mathrm{K}^{+}\right)$e o cloreto $\left(\mathrm{Cl}^{-}\right)$são os mais abundantes (SHIRREFFS \& Maughan, 1997). A magnitude da perda de eletrólitos depende tanto da taxa de sudorese, quanto da composição do suor e ambas variam consideravelmente entre os indivíduos (MAUGHAN et al., 2007).

O Colégio Americano de Medicina do Esporte (ACSM, 2007) recomenda que o consumo de líquidos deve ser próximo ao total de líquidos perdido durante o exercício na forma de suor. Em algumas situaçôes e ambientes, entretanto, tal abordagem não é possível (Coyle, 2004). Apesar da grande variedade de protocolos de reidratação existentes, muitos atletas 
finalizam o período de exercício em um estado de hipoidratação (Ferreira, Alves, Costa, Santana \& Marins, 2010; Mara, Lemos, Brochi, Rohlfs \& Carvalho, 2007; Shirreffs, Aragon-Vargas, KeIL, LOVE \& PHILliPs, 2007). Embora as concentrações de eletrólitos no organismo sejam reguladas com precisão, alteraçōes hematológicas após o exercício (por exemplo, a ocorrência de hiponatremia), são bem documentadas. A hiponatremia, concentração de sódio plasmático menor que $135 \mathrm{mEq} \cdot \mathrm{L}^{-1}$, associada ao exercício ocorre comumente em indivíduos engajados em atividades físicas extenuantes. Como fator etiológico, pode-se citar o consumo de fluidos hipotônicos, com perda excessiva de fluidos pelo suor e urina (DAVIS, VIDEEN, Marino, Vilke, Dunford, Van Camp \& Maharan, 2001; SPEEDy, NOAKES \& SCHNEIDER, 2001).

Poucos trabalhos (Morgan, Patterson \& Nimo, 2004; SaAt, Sirisinghe, Rabindarjeet \& TOSHIHARA, 2005) foram realizados com o objetivo

\section{Materiais e métodos}

\section{Amostra}

Doze atletas do sexo masculino participaram como voluntários neste estudo (idade $=29 \pm 8$ anos, estatura $=1,74 \pm 0,1 \mathrm{~m}$ e massa corporal $=69,5 \pm$ $6,7 \mathrm{~kg}$ ). A coleta foi realizada durante uma competição de duatlo que ocorreu no mês de dezembro - verão brasileiro - em Sapiranga, RS. A competição constituiu-se de $6 \mathrm{~km}$ de corrida, seguidos de $26 \mathrm{~km}$ de ciclismo, e por fim, mais $4 \mathrm{~km}$ de corrida. Por se tratar de um duatlo terrestre, não houve a etapa de natação nesta competição. A prova foi realizada no turno da tarde, iniciando às $13 \mathrm{~h} \mathrm{e} 45 \mathrm{~min}$. No dia da competição, a temperatura ambiente no local da prova às $15 \mathrm{~h}$ era de $31,2^{\circ} \mathrm{C}$ com umidade relativa do ar de 51\%. A determinação da temperatura e umidade relativa do ar foi obtida através de consulta ao INMET (Instituto Nacional de Meteorologia).

\section{Coleta de dados}

O estudo foi aprovado pelo Comitê de Ética em Pesquisa da Universidade Federal do Rio Grande do Sul (protocolo $\mathrm{n}^{\circ} 2006550$ ). Todos os participantes foram informados do objetivo do estudo e assinaram um termo de consentimento livre e esclarecido. Foram coletadas amostras sanguíneas imediatamente antes do início e após o término da de descrever as concentraçôes de $\mathrm{Na}^{+}, \mathrm{Cl}^{-}$e $\mathrm{K}^{+}$no suor e no sangue em resposta ao exercício. A quase totalidade dos estudos foi realizada em laboratório (Morgan, Patterson \& Nimo, 2004; SaAt et al., 2005). Avaliar as respostas fisiológicas de atletas durante suas atividades esportivas competitivas reais são de suma importância, pois permite a obtenção de dados em uma situação em que os indivíduos estão submetidos a uma série de adversidades as quais muitas vezes não podem ser reproduzidas em laboratório. Além disso, a literatura carece de informaçôes sobre concentraçôes de eletrólitos tanto no suor quanto no sangue, assim como dados de balanço de fluidos em atletas praticantes de duatlo. Neste sentido, o objetivo deste estudo foi verificar os níveis de hidratação e as perdas de $\mathrm{Na}^{+}, \mathrm{K}^{+}$e $\mathrm{Cl}^{-}$, analisando a composição do suor e os níveis sanguíneos destes eletrólitos em atletas durante uma competição de duatlo.

competição (à medida que os atletas iam cruzando a linha de chegada, eram encaminhados para a coleta de sangue). Para a análise dos eletrólitos sanguíneos, foram coletados $10 \mathrm{~mL}$ de sangue em tubos sem anticoagulante. As amostras sanguíneas foram refrigeradas e imediatamente transportadas para análise. As amostras foram centrifugadas (Laborline, Elektra, Brasil) a $2500 \mathrm{rpm}$, a $22{ }^{\circ} \mathrm{C}$ por quatro minutos, para a obtenção do soro. Para a análise sanguínea de $\mathrm{Na}^{+}$e $\mathrm{K}^{+}$foi utilizado o método de fotometria de chama (CELM, FC-180, Brasil), onde foi utilizado $100 \mu \mathrm{L}$ de soro diluído em 9,9 mL de água reagente. Para a análise do $\mathrm{Cl}^{-}$foi utilizado o método de Schales - Schales (Cobas Mira Plus, Roche, Suiça), onde foram utilizados $2 \mu \mathrm{L}$ de soro para $580 \mu \mathrm{L}$ de reagente Labtest (Brasil).

Para a coleta do suor foram utilizados adesivos Tegaderm (3M, modelo 3582), os quais são compostos por um filme transparente de poliuretano, recoberto com um adesivo acrílico resistente à água e um componente absorvente de rayon de viscose e polietileno. Desta maneira formam uma película impermeável e estéril capaz de absorver e manter toda a secreção produzida no local de fixação. Os adesivos foram aplicados na superfície da pele em ambos os lados da região das escápulas, imediatamente antes do início da prova. Antes dos adesivos serem aplicados, a pele foi completamente higienizada 
com água deionizada e seca com gazes esterilizadas livres de eletrólitos. Os adesivos foram removidos logo após o término da competição com o auxílio de uma pinça e imediatamente pressionados em uma seringa que expeliu o suor para um micro-tubo. As amostras de suor coletadas foram transportadas sob refrigeração e, então, congeladas para serem posteriormente analisadas. A análise da composição do suor foi realizada pelo método de seletor de íons (Cobas Integra, Roche, Suiça). Para esta análise o suor foi diluído em uma proporção 2:1 de diluente (ref. BP0344) e suor.

A massa corporal foi mensurada antes do início da prova (após os atletas terem esvaziado a bexiga) e ao término da mesma, utilizando-se a balança eletrônica, (Techline, modelo Tec 30), com resolução de 100 g. Para a determinação da quantidade de líquidos ingeridos, as garrafas de cada atleta foram pesadas antes e após o término da competição, por meio de balança eletrônica, (Plenna, modelo MEA-00011) com capacidade máxima para $2 \mathrm{~kg}$ e resolução de 1 g. Todos os atletas foram instruídos: a não cuspirem a água de suas garrafas; a não derramarem água sobre o corpo; e a informarem ao final da competição se beberam alguma água, além daquela contida em suas garrafas fornecidas pelos pesquisadores, ao longo da prova. A água extra consumida pelos atletas era disponibilizada pela organização da competição, na forma de copos de água mineral [composição: $\mathrm{Na}^{+}$ (15,2 mg), $\mathrm{K}^{+}(0,08 \mathrm{mg})$ e $\mathrm{Cl}^{-}$(traços) para cada copo de $200 \mathrm{~mL}$ ] e os atletas foram questionados quanto ao número de copos ingeridos. Os atletas também foram instruídos para que ao finalizarem a prova, não bebessem água antes da coleta de sangue e da mensuração da massa corporal pós-competição.

\section{Cálculos}

Os cálculos utilizados foram baseados nas mudanças da massa corporal e na mensuração da ingestão de fluidos. A perda de água devido à respiração e a água produzida pelo metabolismo foram contabilizadas como perda de suor, pois são consideradas inexpressivas para exercícios que durem menos de três horas (Cheuvront, Haymes \& SaWKA, 2002). Nenhum atleta urinou durante a prova. A perda de líquido correspondente à urina porventura produzida durante a prova não foi contabilizada. Para obter o percentual de desidratação foi utilizada a diferença entre a massa corporal pré e pós-exercício (kg). O percentual de reposição de líquidos perdidos pelo suor durante a prova foi calculado por meio de uma estimativa de reposição de líquidos durante a competição. Para este cálculo utilizou-se a diferença da massa corporal pré e pós-competição $(\mathrm{kg})$ corrigida pela quantidade de líquidos ingeridos (L).

Para obtenção da taxa média de suor foi utilizada a diferença da massa corporal pré e pós-exercício $(\mathrm{kg})$ corrigida pela quantidade de líquidos ingeridos (L) e de urina excretada (L) - (contabilizada como zero, neste caso) - durante a prova e isto foi dividido pelo tempo de prova (h). Para obtenção do volume total de suor foi usada a diferença da massa corporal pré e pós-exercício $(\mathrm{kg})$, adicionando o volume de líquidos ingeridos (L). Para o cálculo da perda total de eletrólitos foi utilizada a variável de volume total de suor multiplicada pela concentração de eletrólitos (mmol) por litro de suor.

\section{Análise estatística}

Os resultados foram analisados com o auxílio do pacote estatístico "Statistical Package for Social Sciences" (SPSS, 15.0). O nível de significância aceito foi $\mathrm{p}<0,05$. Os dados foram expressos como média \pm desvio padrão. Foi realizado teste t de "Student" para amostras independentes para comparar a concentração de eletrólitos do suor coletado nas duas regiōes corporais (lado direito e esquerdo). Para avaliar as variáveis em estudo antes e depois da competição realizou-se o teste t de "Student" para amostras pareadas. A associação entre algumas variáveis foi verificada por meio do coeficiente do teste de correlação linear Produto Momento de Pearson.

\section{Resultados}

A duração da prova foi de $85,0 \pm 6,5$ minutos. Os dados do balanço de fluidos estão sumarizados na TABELA 1. Houve uma redução significativa da massa corporal ao final da competição $(\mathrm{p}<$ $0,001)$ de $2,1 \pm 0,6 \mathrm{~kg}$ (variando entre $1,0-2,9$ $\mathrm{kg})$. 
TABELA 1 - Balanço de fluidos de atletas em competição de duatlo.

\begin{tabular}{lcc}
\hline \multicolumn{1}{c}{$\mathbf{n}=\mathbf{1 2}$} & Média $\pm \mathbf{d p}$ & Valores mínimos - máximos \\
\hline Desidratação $(\%$ da massa corporal) & $3,0 \pm 0,92$ & $1,41-4,36$ \\
Volume de perda de suor $(\mathrm{L})$ & $2,62 \pm 0,70$ & $1,83-4,15$ \\
Taxa média de suor $\left(\mathrm{L} \cdot \mathrm{h}^{-1}\right)$ & $1,86 \pm 0,56$ & $1,22-3,07$ \\
Ingestão de líquidos $(\mathrm{mL})$ & $575,00 \pm 250,10$ & $274,00-1250,00$ \\
\hline
\end{tabular}

Os dados relativos ao suor contam com 11 indivíduos, devido à perda dos adesivos de um atleta durante a competição. Não houve correlação significativa $(r=0,210, p=0,513)$ entre o volume de perda de suor e o volume de líquido consumido durante a prova. Os níveis de eletrólitos no suor estão descritos na TABELA 2. Verificou-se que não houve diferença na concentração de $\mathrm{Na}^{+}, \mathrm{K}^{+} \mathrm{e} \mathrm{Cl}^{-}$ no suor coletado de cada região, assim utilizou-se o valor médio obtido entre os dois locais.

Os dados de eletrólitos sanguíneos estão sumarizados na TABELA 3. Não houve diferença significativa entre os valores séricos dos eletrólitos $\mathrm{Na}^{+}$, $\mathrm{K}^{+}$e $\mathrm{Cl}^{-}$antes e após a competição.

TABELA 2 - Concentração e perda total de eletrólitos no suor de atletas em competição de duatlo.

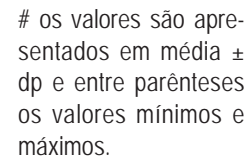

\# os valores são apresentados em média \pm $d p$ e entre parênteses os valores mínimos e máximos.

\begin{tabular}{lccc}
\hline \multicolumn{1}{c}{$\mathbf{n}=\mathbf{1 1}$} & Sódio & Potássio & Cloreto \\
\hline Concentração de eletrólitos $\left(\mathrm{mmol} \cdot \mathrm{L}^{-1}\right)$ & $71,00 \pm 26,05$ & $5,43 \pm 1,98$ & $58,93 \pm 25,99$ \\
& $(34,00$ a 114,00$)$ & $(3,41$ a 8,3$)$ & $(20,25$ a 101,00$)$ \\
Perda total de eletrólitos $(\mathrm{mmol})$ & $132,11 \pm 62,82$ & $10,09 \pm 5,01$ & $109,75 \pm 58,49$ \\
& $(44,41$ a 250,54$)$ & $(4,79$ a 22,74) & $(29,61$ a 221,33) \\
\hline
\end{tabular}

TABELA 3 - Concentração de eletrólitos séricos de atletas em competição de duatlo.

\begin{tabular}{cccc}
\hline $\mathbf{N}=\mathbf{1 2}$ & Antes & Depois & $\Delta$ \\
\hline Sódio $\left(\mathrm{mmol} \cdot \mathrm{L}^{-1}\right)$ & $141,83 \pm 1,99$ & $141,00 \pm 1,81$ & $0,83 \pm 1,80$ \\
& $(138,00-144,00)$ & $(138,00-144,00)$ & \\
Potássio $\left(\mathrm{mmol} \cdot \mathrm{L}^{-1}\right)$ & $4,34 \pm 0,39$ & $4,49 \pm 0,21$ & $0,15 \pm 0,28$ \\
& $(3,70-5,10)$ & $(4,20-5,00)$ & \\
Cloreto $\left(\mathrm{mmol} \cdot \mathrm{L}^{-1}\right)$ & $95,08 \pm 4,32$ & $99,17 \pm 7,11$ & $4,08 \pm 8,81$ \\
& $(87,00-104,00)$ & $(90,00-115,00)$ & \\
\hline
\end{tabular}

\section{Discussão}

Este trabalho foi desenhado para verificar os níveis de hidratação e as concentraçôes de $\mathrm{Na}^{+}, \mathrm{K}^{+}$e $\mathrm{Cl}^{-}$no suor e no sangue de atletas durante uma competição de duatlo terrestre. Os principais achados deste estudo foram que os atletas de duatlo apresentaram um elevado grau de desidratação e não ingeriram quantidade suficiente de líquidos durante a competição. Foram observadas perdas de peso significativas que variaram de 1,0 a 2,9 kg, sendo a média 2,1 kg. Estes valores estão de acordo com um estudo realizado em atletas de corrida em exercício com duração de cerca de uma hora (White, Pomfret, Rennie, Gissane, Wong \& Ford, 1998). No entanto, estão acima da média encontrada em outros trabalhos (FOWKEs GODEK, BartolozZI, BurkHOLDER, SUGARMAN \& DORSHIMER, 2006; FowKes GODEK, BARTOLOZZI \& GODEK, 2005; MAO, Chen \& Ko, 2001). Dois fatores importantes podem ter contribuído para a redução significativa $\mathrm{da}$ massa corporal e consequente desidratação dos atletas: a elevada taxa de sudorese e a ingestáo insuficiente de líquidos. A taxa de sudorese encontrada neste estudo pode ser considerada elevada quando comparada 
a outros estudos da literatura realizados tanto em laboratório (COCHRANE \& SLEIVERT, 1999; INBAR, Morris, Epstein \& Gass, 2004; Morgan, Patterson \& Nimo, 2004) quanto em campo (ShirrefFs, Aragon Vargas, Chamorro, Maughan, Serratosa \& ZACHWIEJA, 2005) e ter contribuído para a perda de peso dos indivíduos. Além disto, apenas cerca de $30 \%$ do volume de suor perdido pelos atletas durante a prova foi reposto através de líquidos consumidos na competição. A percepção da necessidade de reposição de líquidos (sede) parece não ter sido suficiente para garantir uma adequada ingestão de fluidos por estes atletas, já que não houve relação significativa entre a taxa de sudorese e o volume de líquidos ingeridos. Entretanto, alguns fatores podem ter limitado a ingestão de líquidos pelos atletas durante a competição. Beber grandes volumes de líquido pode prejudicar o ritmo de prova dos participantes e levar a um desconforto causado pelo enchimento do estômago (Leiper, Nicholas, Ali, Williams \& Maughan, 2005; Van Nieuwenhoven, VRiens, Brummer \& Brouns, 2000). Sendo assim, muitos atletas podem ter optado por não beber muito líquido, mesmo que estivessem com sede. A ingestão de líquidos durante a prova foi cerca de $575 \mathrm{~mL}$, o que está abaixo da média de consumo observada em outros estudos, nos quais foram realizados exercícios aeróbicos, com intensidade e duração similares ao do presente estudo (BERGERON, 2003; Fowkes GodeK, Bartolozzi \& GodeK, 2005; SHIRREFFs et al., 2005; WhITE et al., 1998). Em uma simulação de competição de duatlo realizada com atletas da categoria júnior, também foi observada ingestão insuficiente de líquidos pelos indivíduos durante o experimento (Iuliano, Naughton, Collier \& Carlson, 1998). O duatlo pode ser considerado um exercício intenso e o intervalo de tempo para a transição entre as suas fases, corrida e ciclismo, é muito curto. Estes fatores podem desfavorecer as oportunidades para ingestão de líquidos no decorrer da competição. Assim, a baixa ingestão de líquidos parece ser uma tendência entre os praticantes de duatlo.

O nível de desidratação dos atletas variou ao final da competição entre 1,41 e 4,36\% da massa corporal pré-competição. Está bem reconhecido que o exercício aeróbico é adversamente afetado pelo calor e pela hipoidratação, resultando em efeitos adversos para os sistemas cardiovascular e termorregulatório (MAUGHAN, Watson \& ShirrefFs, 2007; SAWKa \& Montain, 2000; SHIRreFFs, 2005). Seis dos 12 competidores avaliados apresentaram nível de desidratação superior a 3\%, o que tem sido consistentemente demonstrado ser prejudicial ao desempenho aeróbio (SocIEDADE
Brasileira de Medicina do Esporte, 2009). Além do volume de líquidos ingerido durante a prova, a taxa de sudorese dos atletas também pode ter contribuído para a ampla diferença na taxa de desidratação verificada entre os participantes.

Houve uma grande variação na taxa de sudorese encontrada em nosso estudo, visto que os valores variaram largamente de 1,22 a 3,07 $\mathrm{L} \cdot \mathrm{h}^{-1}$. A taxa média de sudorese encontrada $\left(1,86 \mathrm{~L} \cdot \mathrm{h}^{-1}\right)$ está acima da média verificada em outros trabalhos (Cochrane \& Sleivert, 1999; Inbar et al., 2004; Morgan, Patterson \& Nimo, 2004; ShirrefFs et al., 2005), mas está de acordo com a taxa de sudorese encontrada em um estudo realizado por Fowkes GodeK, Bartolozzi e GodeK (2005), com atletas de "Cross Country", que realizaram uma hora de treino de corrida em ambiente quente, no período da tarde. É importante ressaltar que no trabalho citado previamente, a urina pós-exercício foi aferida e contabilizada como perda de suor, diferentemente de nosso estudo. Contudo, para atletas que realizam exercícios de alta intensidade no calor, taxas de sudorese médias entre 1,0 a $2,5{\mathrm{~L} \cdot \mathrm{h}^{-1}}^{-1}$ são reportadas na literatura (SAWKA \& Montain, 2000). Características individuais como peso corporal, grau de aclimatação, predisposição genética e nível de condicionamento físico também influenciam a resposta de sudorese (ACSM, 2007). Além disto, eventos esportivos também expõem os atletas a uma variedade de fatores que influenciam as perdas de suor, tais como a intensidade e a duração do exercício, as características ambientais e o período do dia em que a competição é realizada (MAUGHAN, Shirreffs, Merson \& Horswill, 2005).

O dia da competição caracterizou-se pela presença de sol com algumas nuvens e por elevada temperatura ambiente $\left(31,2{ }^{\circ} \mathrm{C}\right)$, fatores importantes de estresse térmico no indivíduo ativo (ACSM, 2007). Deve-se considerar, também, que a prova ocorreu no turno da tarde, período do dia em que o padrão de mudança climática é menos favorável para a termorregulação durante o exercício aeróbico. No início do exercício, o gradiente térmico de troca de calor entre a pele e o ambiente pode ter sido menor devido à elevada temperatura ambiente, reduzindo as perdas de calor secas por convecção, condução e radiação. Estas propriedades biofísicas de trocas de calor entre o corpo e o ambiente podem ter influenciado a taxa de sudorese, fazendo com que as perdas de calor por evaporação fossem ativadas mais precocemente e a sudorese se tornasse mais profusa como uma forma compensatória (Cochrane \& SLEIVERT, 1999). 
Estudos mostram que, de fato, indivíduos apresentam uma maior taxa de sudorese no período da tarde em relação ao período da manhã ao realizarem o mesmo exercício físico (Cochrane \& SLeIVERT, 1999; FowKes GodeK, BartolozZI \& GodeK, 2005).

Apesar de o grau de aclimatação não ter sido controlado, os atletas deste estudo estavam acostumados a treinar e a correr no calor. Indivíduos aclimatados têm uma maior eficácia no controle da temperatura corporal e um melhor desempenho físico em ambiente quente. As glândulas sudoríparas écrinas se hipertrofiam e a produção de suor aumenta, possibilitando maior perda de calor por evaporação (GRANTHAM, Cheung, Connes, Febbraio, Gaoua, GonzalezAlonso, Hue, Johnson, Maughan, Meeusen, Nybo, Racinais, ShirrefFs \& DvoraK, 2010; Shibasaki, Wilson \& Crandall, 2006). A aclimatação protege o atleta, mas o benefício termorregulatório obtido através desta adaptação fisiológica ao exercício no calor somente ocorre se o indivíduo mantém-se hidratado durante todo o exercício (ACSM, 2007). Além disto, quanto maior a intensidade do exercício, maior é a produção de calor metabólico, sendo necessário que ocorra a perda deste calor de maneira mais eficaz, através do aumento da dissipação de calor por evaporação do suor, aumentando consequentemente a taxa de sudorese (MAUGHAN et al., 2007).

A composição de eletrólitos do suor também varia entre os indivíduos (ACSM, 2007). As $\left[\mathrm{Na}^{+}\right],[\mathrm{Cl}-]$ e $\left[\mathrm{K}^{+}\right]$no suor dos atletas deste estudo (TABELA 2) estão dentro do intervalo fisiológico de normalidade (ShirRefFs \& MAUGHAN, 1997). Porém, estão acima da média encontrada em outros trabalhos (BERgERON, 2003; MAUGHAN, Merson, Broad \& Shirreffs, 2004; Maughan $\&$ Shirreffs, 2005; SaAT et al., 2005; ShirrefFs et al., 2005). A concentração de eletrólitos no suor é afetada pela taxa de sudorese porque a capacidade de reabsorção ductal das glândulas sudoríparas écrinas, por ser um processo ativo, é limitada e a quantidade de eletrólitos que escapa da reabsorção aumenta com o aumento do fluxo de suor no ducto (SAat et al., 2005; Shamsuddin, Yanagimoto, Kuwahara, Zhang, Nomura \& Kondo, 2005). A hipertonicidade induzida pela desidratação resulta em uma elevada concentração de eletrólitos nos fluidos extracelulares (Morgan, PATterson \& Nimo, 2004). Assim, exercícios intensos e temperaturas altas induzem a uma elevada taxa de sudorese e à desidratação. A desidratação leva a um aumento dos níveis de eletrólitos nas glândulas sudoríparas écrinas, enquanto a elevada taxa de sudorese induz a uma menor reabsorção dos eletrólitos que escaparam para o suor. Portanto, isto pode explicar a elevada $\left[\mathrm{Na}^{+}\right],\left[\mathrm{Cl}^{-}\right]$e $\left[\mathrm{K}^{+}\right]$no suor dos atletas, pois, de fato, os indivíduos estavam hipoidratados e tiveram uma taxa de sudorese que pode ser considerada elevada. Consequentemente, isto também levou a elevadas perdas totais de $\mathrm{Na}^{+}, \mathrm{K}^{+}$e Cl- pelo suor $(132 \mathrm{mmol}$, $10 \mathrm{mmol}$ e $109 \mathrm{mmol}$, respectivamente).

Embora não tenha sido feita a correção do volume plasmático, as $\left[\mathrm{Na}^{+}\right],[\mathrm{Cl}]$ e $\left[\mathrm{K}^{+}\right]$séricas (TABELA 3) mantiveram-se praticamente estáveis durante os 85 minutos de realização da prova. Resultados semelhantes também foram observados em outros trabalhos. Um estudo mostrou que após duas horas de corrida em esteira não houve mudanças nas $\left[\mathrm{Na}^{+}\right] \mathrm{e}\left[\mathrm{K}^{+}\right]$sanguíneas (Cochrane \& Sleivert, 1999). Outro trabalho também verificou que, após 40 minutos de exercício em cicloergômetro, não foram encontradas diferenças significativas nas $\left[\mathrm{Na}^{+}\right],[\mathrm{Cl}]$ e $\left[\mathrm{K}^{+}\right]$séricas (SAAT et al., 2005). No estado hipohidratado há uma considerável redistribuição de água a partir dos espaços intra e extracelulares do tecido muscular e cutâneo, favorecendo, assim, a manutenção do volume plasmático e o conteúdo hídrico de diversos órgãos e sistemas corporais (Nose, Morimoto \& Ogura, 1983).

Os atletas completaram a prova em tempos diferentes, levando-os a apresentar uma variação no tempo de permanência com os adesivos de coleta de suor. Este fato pode ser citado como uma limitação do trabalho. Entretanto, esta diferença de tempo (cerca de seis minutos) levaria a uma variação considerada mínima no volume de suor secretado, o que não seria suficiente para alterar significativamente a composição do suor dos participantes. Além disto, se o tempo para completar a prova tivesse sido pré-estabelecido, é provável que os indivíduos se exercitassem em intensidades diferentes, o que levaria, possivelmente, a diferentes taxas de sudorese e concentrações de eletrólitos no suor. Outro ponto relevante de mencionar seria que o volume de urina não foi levado em consideração para o cálculo do volume total de suor. $\mathrm{O}$ volume diário de urina varia entre os indivíduos, mas é tipicamente entre 1 e $2 \mathrm{~L} /$ dia. Isto corresponde a uma formação média de urina de 40 a $80 \mathrm{~mL} / \mathrm{h}$. Entretanto, durante exercícios vigorosos, reduçōes significativas de 20 a $60 \%$ nestas taxas são reportadas. Estas reduçôes se devem principalmente à ação do hormônio antidiurético secretado em resposta ao início do exercício e ao marcante redirecionamento do fluxo sanguíneo renal para a musculatura ativa (ACSM, 2007; MAUGHAN, SHIRREFFS \& LeIPER, 2007; Melin, Koulmann, Jimenez, Savourey, Launay, Cottet-Emard, Pequignot, Allevard \& Gharib, 
2001). Adicionalmente, por se tratar de um estudo de campo, existem dificuldades práticas no controle desta variável (MAUGHAN, SHIRREFFs \& LeIPER, 2007). Desta forma, considera-se que a diferença na massa corporal pré e pós-exercício têm uma boa correlação com o volume de suor perdido (ACSM, 2007; MARA et al., 2007).

No presente estudo, a estimativa de alteração do volume plasmático não foi realizada. Entretanto, de acordo com a revisão sistemática de literatura de Kargotich, Goodman, Keast e Morton (1998) encontramos três modalidades de estudos envolvendo a determinação de parâmetros sanguíneos e a desidratação: 1) estudos que não aferem ou mencionam as alterações no volume plasmático; 2) estudos que aferem as alterações no volume plasmático e não corrigem os resultados; e 3) estudos que aferem as alterações no volume plasmático e corrigem os resultados. Devido ao fato de o presente estudo ter sido realizado em campo, não foi possível estruturar a logística para a determinação do hematócrito e hemoglobina, parâmetros utilizados na estimativa do volume plasmático e, portanto a não aferição do volume plasmático é uma limitação deste estudo. Por outro lado, é possível especular que as perdas de eletrólitos pelo suor possam ter levado a alterações nas concentrações sanguíneas dos eletrólitos, a qual pode não ter sido detectada devido à hemoconcentração causada por possíveis alterações no volume plasmático. Não se descarta a possibilidade desta provável hemoconcentração induzida pela desidratação ser um mecanismo regulador de controle da concentração fisiológica sérica de eletrólitos. Entretanto, estudos futuros são necessários para melhor esclarecer o comportamento do volume plasmático e dos eletrólitos séricos envolvendo protocolos de desidratação e a modalidade de duatlo.

\title{
Conclusões
}

Atletas de duatlo competindo em ambiente quente não ingeriram quantidade suficiente de líquidos para repor suas perdas de suor e muitos deles incorreram com substancial desidratação. Os participantes apresentaram elevada taxa de sudorese acompanhada de perdas de $\mathrm{Na}^{+}, \mathrm{K}^{+}$e $\mathrm{Cl}^{-}$. Apesar disto, não ocorreram alterações nas $\left[\mathrm{Na}^{+}\right],\left[\mathrm{K}^{+}\right] \mathrm{e}$ $\left[\mathrm{Cl}^{-}\right]$séricas.

\begin{abstract}
Electrolyte losses during a land based duatlhon competition in the heat

Prolonged sports events such as duathlon ( $6 \mathrm{~km}$ running, $26 \mathrm{~km}$ cycling and $4 \mathrm{~km}$ running), may lead the athlete to a fluid and electrolyte imbalance, due to high sweat rates, especially in a hot environment. The present study evaluated sodium $\left(\mathrm{Na}^{+}\right)$, potassium $\left(\mathrm{K}^{+}\right)$and chloride $\left(\mathrm{Cl}^{-}\right)$losses during a duathlon competition performed in the heat $\left(31,2{ }^{\circ} \mathrm{C}\right.$ and $51 \%$ relative humidity), analyzing the sweat composition and blood levels of these electrolytes. Twelve athletes took part in this study. Blood samples were obtained pre- and post-exercise and sweat was collected using sweat patches. The average time to complete the competition was $85.0 \pm 6.57 \mathrm{~min}$. The percentage of dehydration was $3.0 \pm 0.92 \%$. The replacement of fluid losses was $31 \pm 18.7 \%$. The sweat rate was $1.86 \pm 0.56{\mathrm{~L} \cdot h^{-1}}^{-}$Sweat $\mathrm{Na}^{+}, \mathrm{K}^{+}$, and $\mathrm{Cl}^{-}$ concentrations were $71 \pm 26.05 \mathrm{mmol} \cdot \mathrm{L}^{-1}, 5.43 \pm 1.98 \mathrm{mmol} \cdot \mathrm{L}^{-1}$ and $58.93 \pm 25.99 \mathrm{mmol} \cdot \mathrm{L}^{-1}$, respectively. The total sweat loss of $\mathrm{Na}^{+}, \mathrm{K}^{+}$and $\mathrm{Cl}^{-}$was $132.11 \pm 62.82 \mathrm{mmol}, 10.09 \pm 5.01 \mathrm{mmol}$ and $109.75 \pm 58.49$ mmol, respectively. In conclusion, the athletes did not drink enough liquid to replace their volume of fluid loss. Furthermore, the participants presented high sweat rate accompanied by losses of $\mathrm{Na}^{+}, \mathrm{K}^{+}$and $\mathrm{Cl}^{-}$. However, serum electrolyte concentrations were not changed.
\end{abstract}

UnITERMS: Exercise; Dehydration; Sweat; Temperature. 


\section{Referências}

AMERICAN COLLEGE OF SPORTS MEDICINE. ACSM's position stand: exercise and fluid replacement. Medicine and Science in Sports and Exercise, Madison, v.39, n.2, p.377-90, 2007.

BERGERON, M.F. Heat cramps: fluid and electrolyte challenges during tennis in the heat. Journal of Science and Medicine in Sport, Belconnen, v.6, n.1, p.19-27, 2003.

CHEUVRONT, S.N.; HAYMES, E.M.; SAWKA, M.N. Comparison of sweat loss estimates for women during prolonged high intensity running. Medicine and Science in Sports and Exercise, Madison, v.34, p.1344-50, 2002.

COCHRANE, D.J.; SLEIVERT, G.G. Do changing patterns of heat and humidity influence thermoregulation and endurance performance? Journal of Science and Medicine in Sport, Belconnen, v.2, n.4, p.322-32, 1999.

COYLE, E.F. Fluid and fuel intake during exercise. Journal of Sports Sciences, London, v.22, p.39-55, 2004.

DAVIS, D.P.; VIDEEN, J.S.; MARINO, A.; VILKE, G.M.; DUNFORD, J.V.; VAN CAMP, S.P.; MAHARAM, L.G. Exercise-associated hyponatremia in marathon runners: a two-year experience. The Journal of Emergency Medicine, New York, v.21, n.1, p.47-57, 2001.

FERREIRA, F.G.; ALVES, K.; COSTA, N.M.B.; SANTANA, A.M.C.; MARINS, J.C.B. Efeito do nível de condicionamento físico e da hidratação oral sobre a homeostase hídrica em exercício aeróbico. Revista Brasileira de Medicina do Esporte, São Paulo, v.16, p.166-70, 2010.

FOWKES GODEK, S.; BARTOLOZZI, A.R.; BURKHOLDER, R.; SUGARMAN, E.; DORSHIMER, G. Core temperature and percentage of dehydration in professional football linemen and backs during preseason practices. Journal of Athletic Training, Dallas, v.41, n.1, p.8-17, 2006.

FOWKES GODEK, S.; BARTOLOZZI, A.R.; GODEK, J.J. Sweat rate and fluid turnover in American football players compared with runners in a hot and humid environment. British Journal of Sports Medicine, London, v.39, p.205-11, 2005. GRANTHAM, J.; CHEUNG, S.S.; CONNES, P.; FEBBRAIO, M.A.; GAOUA, N.; GONZALEZ-ALONSO, J.; HUE, O.; JOHNSON, J.M.; MAUGHAN, R.J.; MEEUSEN, R.; NYBO, L.; RACINAIS, S.; SHIRREFFS, S.M.; DVORAK, J. Position Statement: current knowledge on playing football in hot environments. Scandinavian Journal of Medicine and Science in Sports, Copenhagen, v.20, p.161-67, 2010. Supplement 3.

INBAR, O.; MORRIS, N.; EPSTEIN, Y.; GASS, G. Comparison of thermoregulatory responses to exercise in dry heat among prepuberal boys, young adults and older males. Experimental Physiology, Cambridge, v.86, n.6, p.691-700, 2004. IULIANO, S.; NAUGHTON, G.; COLLIER, G.; CARLSON, J. Examination of the self-selected fluid intake practices by junior athletes during a simulated duathlon event. International Journal of Sports Nutrition, Champaign, v.8, p.10-23, 1998. KARGOTICH, S.; GOODMAN, C.; KEAST, D.; MORTON, A.R. The influence of exercise-induced plasma volume changes on the interpretation of biochemical parameters used for monitoring exercise, training and sport. Sports Medicine, Auckland, v.26, p.101-17, 1998. KENEFIK, R.W.; SAWKA, M.N. Heat exhaustion and dehydration as causes of marathon collapse. Sports Medicine, Auckland, v.37, n.4-5, p.378-81, 2007.

LEIPER, J.B.; NICHOLAS, C.W.; ALI, A.; WILLIAMS, C.; MAUGHAN, R.J. The effect of intermittent high-intensity running on gastric emptying of fluids in man. Medicine and Science in Sports and Exercise, Madison, v.37, p.240-7, 2005. MAO, I.F.; CHEN, M.L.; KO, Y.C. Electrolyte loss in sweat and iodine deficiency in a hot environment. Archives of Environmental Health, Chicago, v.56, n.3, p.271-7, 2001.

MARA, L.S.; LEMOS, R.; BROCHI, L.; ROHLFS, I.C.P.M.; CARVALHO, T. Alteraçōes hidroeletrolíticas agudas ocorridas no Triatlon Ironman Brasil. Revista Brasileira de Medicina do Esporte, São Paulo, v.13, p.397-401, 2007.

MAUGHAN, R.J.; MERSON, S.J.; BROAD, N.P.; SHIRREFFS, S.M. Fluid and electrolyte intake and loss in elite soccer players during training. International Journal of Sports Nutrition and Exercise Metabolism, Champaign, v.14, p.333-46, 2004.

MAUGHAN, R.J.; SHIRREFFS, S. Exercise in the heat: challenges and opportunities. Journal of Sports Sciences, London, v.22, p.917-27, 2004.

MAUGHAN, R.J.; SHIRREFFS, S.M.; LEIPER, J.B. Errors in the estimation of hydration status from changes in body mass. Journal of Sports Sciences, London, v.25, n.7, p.797-804, 2007.

MAUGHAN, R.J.; SHIRREFFS, S.M.; MERSON, S.J.; HORSWILL, C.A. Fluid and electrolyte balance in elite male football (soccer) players training in a cool environment. Journal of Sports Sciences, London, v.23, p.73-9, 2005.

MAUGHAN, R.J.; WATSON, P.; EVANS, G.H.; BROAD, N.; SHIRREFFS, S.M. Water Balance and Salt Losses in Competitive Football. International Journal of Sports Nutrition and Exercise Metabolism, Champaign, v.17, p.583-94, 2007. MAUGHAN, R.J.; WATSON, P.; SHIRREFFS, S.M. Heat and cold: what does the environment do to the marathon runner? Sports Medicine, Auckland, v.37, n.4-5, p.396-9, 2007. 
MELIN, B.; KOULMANN, N.; JIMENEZ, C.; SAVOUREY, G.; LAUNAY, J.C.; COTTET-EMARD, J.M.; PEQUIGNOT, J.M.; ALLEVARD, A.M.; GHARIB, C. Comparison of passive heat or exercise-induced dehydration on renal water and electrolyte excretion: the hormonal involvement. European Journal of Applied Physiology, Berlin, v.85, p.250-8, 2001. MORGAN, R.M.; PATTERSON, M.J.; NIMMO, M.A. Acute effect of dehydration on sweat composition in men during prolonged exercise in the heat. Acta Physiologica Scandinavica, Stcockholm, v.182, p.37-43, 2004.

NOSE, H.; MORIMOTO, T.; OGURA, K. Distribution of water losses among fluid compartments of tissues under thermal dehydration in the rat. The Japanese Jornal of Physiology, Tokyo, v.33, p.1019-29, 1983.

SAAT, M.; SIRISINGHE, R.G.; RABINDARJEET, S.; TOSHIHARA, Y. Effects of short-term exercise in the heat on thermoregulation, blood parameters, sweat secretion and sweat composition of topic-dwelling subjects. Journal of Physiological Anthropology and Applied Human Science, Tokyo, v.24, n.5, p.541-9, 2005.

SAWKA, M.N.; MONTAIN, S. Fluid and electrolyte supplementation for exercise heat stress. American Journal of Clinical Nutrition, Bethesda, v.72, p.564-72, 2000. Supplement.

SHAMSUDDIN, A.K.M.; YANAGIMOTO, S.; KUWAHARA, T.; ZHANG, Y.; NOMURA, C.; KONDO, N. Changes in the index sweat ion concentration with increasing sweat during passive heat stress in humans. European Journal of Applied Physiology, Berlin, v.94, p.292-7, 2005.

SHIBASAKI, M.; WILSON, T.E.; CRANDALL, C.G. Neural control and mechanisms of eccrine sweating during heat stress and exercise. Journal of Applied Physiology, Washington, v.100, p.1692-1701, 2006.

SHIRREFFS, S.M. The importance of good hydration for work and exercise performance. Nutrition Reviews, Baltimore, v.63, n.6-2, p.S14-21, 2005.

SHIRREFFS, S.M.; ARAGON VARGAS, L.F.; CHAMORRO, M.; MAUGHAN, R.J.; SERRATOSA, L.; ZACHWIEJA, J.J. The sweating response of elite professional soccer players to training in the heat. International Journal of Sports Medicine, Stuttgard, v.26, p.90-5, 2005.

SHIRREFFS, S.M.; ARAGON-VARGAS, L.F.; KEIL, M.; LOVE; T.D.; PHILLIPS, S. Rehydration after exercise in the heat: a comparison of 4 commonly used drinks. International Journal of Sports Nutrition and Exercise Metabolism, Champaign, v.17, p.244-58, 2007. SHIRREFFS, S.M.; MAUGHAN, R.J. Whole body sweat collection in man: an improved method with some preliminary data on electrolyte composition. Journal of Applied Physiology, Washington, v.82, p.336-41, 1997.

SOCIEDADE BRASILEIRA DE MEDICINA DO ESPORTE (SBME). Diretriz da Sociedade Brasileira de Medicina do Esporte: modificações dietéticas, reposição hídrica, suplementos alimentares e drogas: comprovação da ação ergogênica e potenciais riscos para a saúde. Revista Brasileira de Medicina do Esporte, São Paulo, v.15, n.3, p.1-11, 2009.

SPEEDY, D.B.; NOAKES, T.D.; SCHNEIDER, C. Exercise associated hyponatremia: a review. Emergency Medicine, New York, v.13, p.17-27, 2001.

VAN NIEUWENHOVEN, M.A.; VRIENS, B.E.; BRUMMER, R.J.; BROUNS, F. Effect of dehydration on gastrointestinal function at rest and during exercise in humans. European Journal of Applied Physiology, Berlin, v.83, p.578-84, 2000. WHITE, J.A.; POMFRET, D.K.; RENNIE, S.; GISSANE, C.; WONG, J.; FORD, M. Fluid replacement needs of welltrained male and female athletes during indoor and outdoor steady state running. Journal of Science and Medicine in Sport, Belconnen, v.1, n.3, p.131-42, 1998.

\section{Agradecimentos}

À Federação Gaúcha de Triathlon pelo apoio durante a competição. À Jocelito Martins e Otávio Bertoletti pelo apoio técnico.

ENDEREÇO

Geórgia Franco Becker

Escola de Educação Física

Universidade Federal do Rio Grande do Sul

R. Felizardo, 750

90690-200 - Porto Alegre - RS - BRASIL e-mail: georgia.becker@gmail.com

Recebido para publicação: 07/ 02/ 2010

1a. Revisão: 03/ 02/2011

2a. Revisão: 21/ 02/ 2011

Aceito: 25/02/2011 